6 Nolan CM, Goldberg SV, Buskin SE. Hepatotoxicity associated with isoniazid preventive therapy: a 7-year survey from a public health tuberculosis clinic. JAMA 1999; 281: 1014-1018.

7 Grant AD, Mngadi KT, van Halsema CL, et al. Adverse events with isoniazid preventive therapy: experience from a large trial. AIDS 2010; 24: Suppl. 5, S29-S36.

8 Snider DEJr, Caras GJ. Isoniazid-associated hepatitis deaths: a review of available information. Am Rev Respir Dis 1992; 145: 494-497.

9 World Health Organization. Guidelines on the Management of Latent Tuberculosis Infection. Geneva, World Health Organization, 2014. Available from: www.who.int/tb/publications/ltbi_document_page/en/

10 Moher D, Liberati A, Tetzlaff J, et al. Preferred reporting items for systematic reviews and meta-analyses: the PRISMA statement. PLoS Med 2009; 6: e1000097.

11 Public Health Agency of Canada. Canadian Tuberculosis Standards 7th Edition 2013. 2014. Ottawa, ON, Centre for Communicable Diseases and Infection Control, Public Health Agency of Canada, 2014. Available from: www. respiratoryguidelines.ca/tb-standards-2013

12 Centers for Disease Control and Prevention. Latent Tuberculosis Infection: A Guide for Primary Health Care Providers. Atlanta, GA, Centers for Disease Control and Prevention, 2013. Available from: www.cdc.gov/tb/ publications/ltbi/pdf/TargetedLTBI.pdf

13 Saukkonen JJ, Cohn DL, Jasmer RM, et al. An official ATS statement: hepatotoxicity of antituberculosis therapy. Am J Respir Crit Care Med 2006; 174: 935-952.

14 Haut Conseil de la Santé Publique. Enquête Autour d'un Cas de Tuberculose: Recommandations Pratiques. [SurveyAround a Case of Tuberculosis Practical Recommendations]. Paris, Haut Conseil de la santé publique, 2013. Available from: www.hcsp.fr/Explore.cgi/Telecharger?NomFichier=hcspr20131025_enquetecastuberculoserecoprat.pdf

15 Sociedade Portuguesa de Pneumologia. Tratamento da Tuberculose Latente. Revisão Das Normas [Treatment of Latent Tuberculosis. Review of Rules]. Lisbon, Sociedade Portuguesa de Pneumologia, 2006. Available from: www. dgs.pt/documentos-e-publicacoes/tratamento-da-tuberculose-latente.aspx

16 Socialsyrelson. Rekommendationer för preventiva insatser mot tuberkulos - hälsokontroll, smittspårning och vaccination [Recommendations for preventive measures against tuberculosis - health surveillance, contact tracing and vaccination]. www.socialstyrelsen.se/smittskydd/sjukdomar/smittsammasjukdomarochsmittamnen/tuberkulos Date last accessed: November 11, 2014. Date Last updated: March 01, 2012.

17 Health Protection Surveillance Centre. Guidelines on the Prevention and Control of Tuberculosis in Ireland 2010 Dublin, Health Protection Surveillance Centre, 2010. Available from: www.hpsc.ie/AboutHPSC/ScientificCommittees/ Publications/File,4349,en.pdf

\title{
A novel whole-blood miRNA signature for a rapid diagnosis of pulmonary tuberculosis
}

To the Editor:

One essential aspect for controlling the spread of tuberculosis (TB) is to diagnose it in an early stage. However, the commonly used test systems are still insufficient. Moreover, current assays perform poorly in discriminating between active TB and latent tuberculosis infection (LTBI). Due to limited knowledge of promising TB biomarkers, global "omics" approaches bear attractive options to follow [1]. MiRNAs are important post-transcriptional regulators shown to be involved in the modulation of immune responses against intracellular pathogens [2]. These findings open the possibility of using miRNAs as TB biomarkers for diagnosis. In the present study, we have investigated their role as blood biomarkers for detecting active TB.

Blood samples were collected from five different participating institutions located in Barcelona (Spain). We enrolled a total of 50 individuals, classified into three groups: 1) 17 LTBI individuals. They had been in close recent contact with a high exposure to a smear positive or negative pulmonary TB ( $\mathrm{pTB}$ ) patient. The inclusion criteria were a positive tuberculin skin test (TST), at least one positive result for one of the interferon (IFN)- $\gamma$ assays and no more than 2 weeks of chemoprophylaxis. 2) 17 active pTB patients with a positive culture for Mycobacterium tuberculosis and no more than 2 weeks of anti-TB therapy. 3) 16 

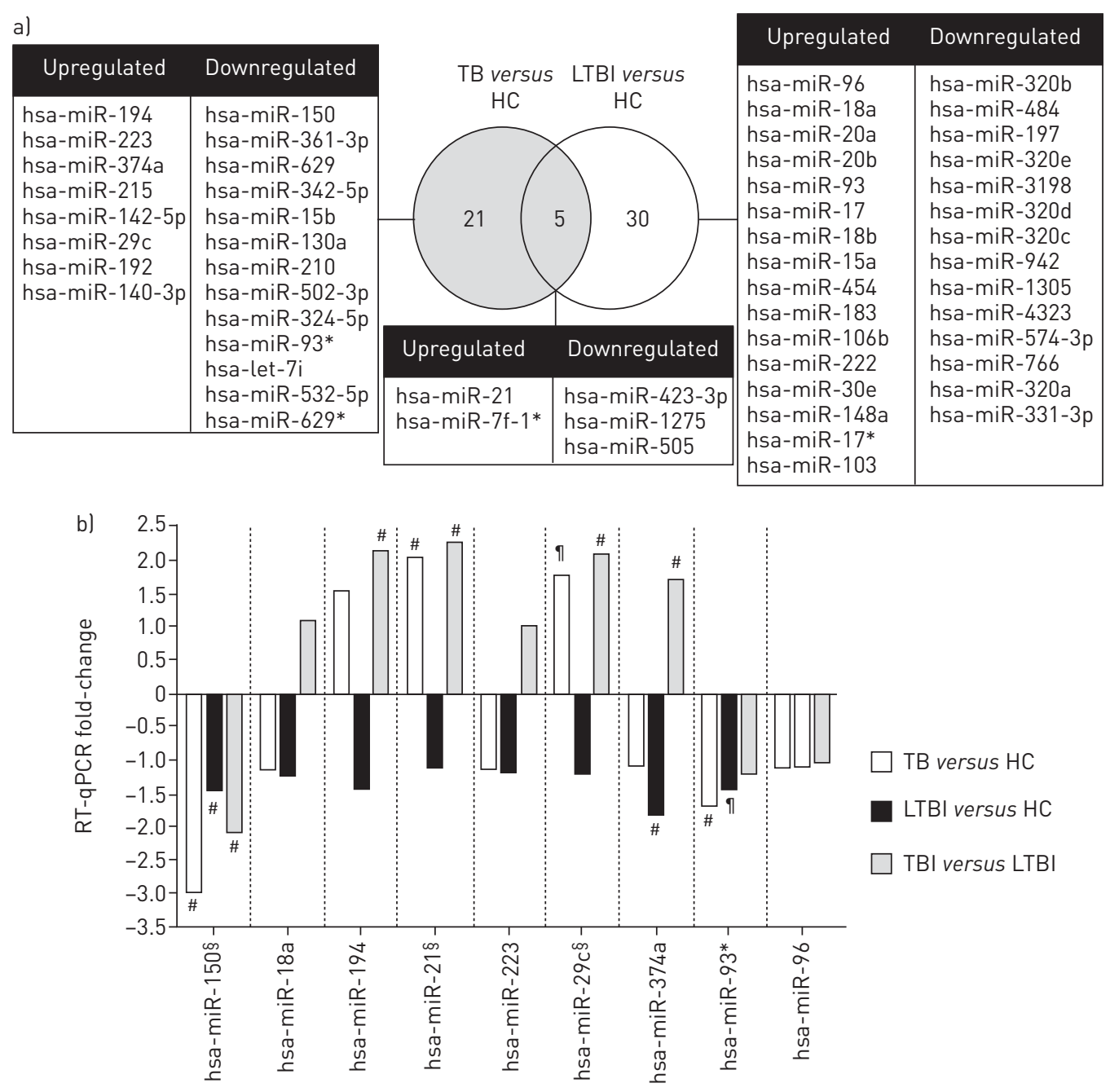

FIGURE 1 Differentially deregulated miRNAs in active tuberculosis (TB) and latent tuberculosis infected (LTBI) individuals with respect to healthy controls (HC). a) Venn diagram of upregulated and downregulated miRNAs. An unpaired two-tailed t-test was applied to evaluate statistical differences in miRNA expression between groups. For further analysis, miRNAs were selected by the following criteria, i) an unadjusted p-value $<0.05$ for the comparisons, ii) detection in all samples of at least one group of each comparison, and iii) upregulation or downregulation with a fold-change $>1.3$. Microarray data were $\log$-transformed and quantile normalisation was applied to normalise expression values using the freely available $\mathrm{R}$ software. For this analysis, a total of 25 samples were tested representing nine pulmonary active TB patients, 10 individuals with LTBI and six healthy controls. They were randomly picked from all available samples excluding patients with comorbidities. b) Relative fold-change of miRNA transcripts analysed by RT-qPCR from different patient groups and controls. Nine selected miRNAs were analysed by RT-qPCR with the following selection criteria: i) a strong fold-change deregulation and/or ii) relevance as suggested in the literature. Data represents the mean fold-change of each miRNA validated by RT-qPCR for TB versus HC (white bars), LTBI versus HC (black bars) and TB versus LTBI (grey bars). The fold-change calculated for each miRNA indicates downregulation or upregulation when the value is negative or positive, respectively. We used two endogenous controls for normalisation of threshold cycle (Ct) values in samples (snoRNA RNU48 (official symbol: SNORD48) and snRNA RNU6B (official symbol: RNU6-2)). All samples were run in duplicates. Fold-change values were computed by using the $2^{-\Delta \Lambda C t}$ method. Validation of differentially-expressed miRNAs by RT-qPCR was assessed with the samples from the complete study group covering 17 pulmonary active TB patients, 17 individuals with LTBI and 16 healthy controls. Five patients with active TB presented the following comorbidities: i) chronic obstructive pulmonary disease (COPD) and lung tumour, ii) hepatitis C virus (HCV) and hepatitis B virus (HBV) infection, iii) follicular cyst, iv) urothelial carcinoma and v) schizophrenia. TB: tuberculosis; HC: healthy controls; LTBI: latent tuberculosis infection; RT-qPCR: Real time-quantitative polymerase chain reaction. ${ }^{\#}: \mathrm{p}<0.05{ }^{\text {ๆ}: ~} \mathrm{p}=0.06{ }^{\S}$ : significantly deregulated miRNAs in TB versus $\mathrm{HC}$ and TB versus LTBI comparisons.

healthy individuals with negative TST and negative IFN- $\gamma$ assay results. They were healthcare workers with no risk of TB exposure and recruited in the course of routine examinations that excluded comorbidities (further details on patient's characteristics can be provided by the authors upon request).

Written informed consent was obtained from all study participants. The corresponding Ethics Committees provided ethics approval. A total of $2.5 \mathrm{~mL}$ of blood was extracted in PAXgene Blood RNA tubes (PreAnalytiX, Hombrechtikon, Switzerland). Total RNA was isolated with a PAXgene Blood miRNA kit (Qiagen, Hilden, Germany). Microarray analysis was performed as described [3] using SurePrint G3 $8 \times 60 \mathrm{~K}$ miRNA microarrays (Agilent Technologies, Santa Clara, California). Microarray results were validated by RT-qPCR using miScript PCR System (Qiagen). 
To identify miRNAs differentially expressed between the three study groups, blood samples were first analysed by microarrays. Differentially deregulated miRNAs in active TB and LTBI individuals with respect to healthy controls (TB versus $\mathrm{HC}$ and LTBI versus $\mathrm{HC}$ ) are detailed in fig. 1a. MiRNA levels in the active TB group compared with controls were 3.27-1.32-fold over-expressed and 2.02-1.31-fold under-expressed, respectively.

Nine selected differentially expressed miRNAs were further analysed by RT-qPCR. Three miRNAs were significantly deregulated in active TB patients with respect to those with LTBI and healthy controls: hsa-miR-150 (down-regulation), hsa-miR-21 (up-regulation) and hsa-miR-29c (up-regulation) (fig. 1b). While most of validated miRNAs showed the same direction of deregulation as in the microarray-based analysis, in some occasions the microarray threshold of detecting expression differences seemed quite low. This enforces the need for RT-qPCR validation of microarray results.

In order to define a miRNA-signature for rapid pTB diagnosis, validated miRNAs with mean fold-change values $>1.5$, and a significant deregulation in the comparisons TB versus LTBI and/or TB versus HC were selected. These were hsa-miR-150, hsa-miR-21, hsa-miR-29c and hsa-miR-194. We then applied a support vector machine with linear kernel (20 repetitions of standard 10-fold cross validation) for computing the accuracy, sensitivity, specificity and positive predictive values (PPV) with the corresponding 95\% confidence intervals. Interestingly, the selected miRNA-signature had a 90.1\% accuracy (89.75-90.45\%), a $91.21 \%$ sensitivity $(90.8-91.62 \%)$, a $87.95 \%$ specificity $(87.37-88.52 \%)$ and a $93.63 \%$ PPV (93.35-93.91\%) for diagnosing active pTB (comparison TB versus LTBI+HC).

Several studies aiming to identify deregulated miRNAs in active TB have been performed; however, only some of them addressed the issue of diagnostic performance [4-8]. The data of these studies revealed that the quantification of a single miRNA is not a reliable indicator of active TB $[4,5]$. Amongst the reasons is the neglect of miRNA expression differences between individuals. When miRNAs were evaluated in combination, then reasonable sensitivities and specificities were reached [7,8]. These latter studies using serum samples identified 6 (miR-378, miR-483-5p, miR-22, miR-29c, miR-101 and miR-320b) and 15 miRNAs (let-7e, miR-146a, miR-148a, miR-16, miR-192, miR-193a-5p, miR-25, miR-365, miR-451, miR-532-5p, miR-590-5p, miR-660, miR-885-5p, miR-223* and miR-30e) respectively, and are in the order of the test performance described here using just 4 miRNAs quantified from whole blood.

It is intriguing to see that different miRNA signatures are being proposed for the diagnosis of active TB. Most studies were composed of two experimental steps, a primary miRNA screen and a subsequent result validation by RT-qPCR. Therefore, the resolution of the primary screen, for example a microarray platform or RNA sequencing, as well as the patient grouping influences the choice of miRNAs to be evaluated further and thus the final miRNA signature [9]. Furthermore, the choice of starting material for miRNA quantification like serum, individually separated blood cell populations or whole blood is expected to affect miRNA signatures.

The observed link between our deregulated miRNA signature and active TB urges to better understand the underlying mechanisms. MA et al. [10] have described that miR-29 suppresses the immune response to intracellular pathogens by targeting IFN- $\gamma$ mRNA. The upregulated miR-21 is involved in the reduction of host T-helper (Th) 1 responses [11]. The third overexpressed miRNA was miR-194. This miRNA together with miR-29 targets components of the Wnt signalling pathway that seems to play a role in $\mathrm{TB}$ pathogenesis $[12,13]$. Finally, miR-150 is under-expressed in active TB. Its primary target is a negative regulator of natural killer (NK) cell maturation. Thus a reduction of mR-150 levels may indicate the development of fewer mature NK cells which are early innate effector cells controlling invading pathogens [14]. Altogether, these findings are in line with the hypothesis that the four deregulated miRNAs can create an immunologically favourable environment for M. tuberculosis expansion.

Limitations of our study need to be addressed. First, the number of patients included is limited and does not allow distinction between ethnic groups. Nonetheless, the newly observed deregulated miRNAs are consistent with previous study results or immunological concepts on TB pathogenesis. Second, the mechanistic link between the observed miRNA signature and active TB remains hypothetical. However, the identified signature together with the functional role of the respective miRNAs directly suggests experiments to move forward in this matter. Third, blood count differences between groups may impact miRNA expression profiles. However, statistical evaluation of such influences reveals that they contribute only partly to a miRNA profile and that they do not significantly affect the feasibility to correlate a miRNA signature with a disease phenotype in humans [15].

Taken together, we here describe a novel, whole blood-derived miRNA signature that enables rapid diagnosis of pTB with $91.21 \%$ sensitivity and $87.95 \%$ specificity. Larger cohort studies are required to validate this signature in extrapulmonary $\mathrm{TB}$ cases and other pulmonary pathologies common in TB differential 
diagnosis. Finally, if the accurate differentiation between infection and disease is validated, it should be possible to build up a simple, rapid and cheap point-of-care test that is urgently needed even today.

0 @ERSpublications

A novel, whole-blood miRNA signature enables to rapidly diagnose TB with $\mathbf{9 1 . 2 1 \%}$ sensitivity and $87.95 \%$ specificity http://ow.ly/FVvHt

Irene Latorre ${ }^{1,2,3,14}$, Petra Leidinger ${ }^{1,14}$, Christina Backes ${ }^{4}$, Jose Domínguez ${ }^{3,5,6}$, Maria Luiza de Souza-Galvão ${ }^{7}$, Jose Maldonado $^{8}$, Cristina Prat ${ }^{3,5,6}$, Juan Ruiz-Manzano ${ }^{3,6,9}$, Francisca Sánchez ${ }^{10}$, Irma Casas ${ }^{6,11}$, Andreas Keller ${ }^{4}$, Hagen von Briesen $^{12}$, Hernando Knobel ${ }^{6,10}$, Eckart Meese ${ }^{1}$ and Andreas Meyerhans, ${ }^{2,13}$

${ }^{1}$ Human Genetics Department, Saarland University, Saarbrücken, Germany. ${ }^{2}$ Infection Biology Laboratory, Department of Experimental and Health Sciences, Universitat Pompeu Fabra, Barcelona, Spain. ${ }^{3}$ CIBER Enfermedades Respiratorias (CIBERES), Instituto de Salud Carlos III, Badalona, Spain. ${ }^{4}$ Clinical Bioinformatics, Saarland University, Saarbrücken, Germany. ${ }^{5}$ Department of Microbiology, Hospital Universitari Germans Trias i Pujol, Institut d'Investigació Germans Trias i Pujol, Badalona, Spain. ${ }^{6}$ Universitat Autònoma de Barcelona, Bellaterra, Spain. ${ }^{7}$ Unitat de Tuberculosis Vall d'Hebron-Drassanes, Barcelona, Spain. ${ }^{8}$ Serveis Clínics de Barcelona, Barcelona, Spain. ${ }^{9}$ Department of Pneumology, Hospital Universitari Germans Trias i Pujol, Institut d'Investigació Germans Trias i Pujol, Badalona, Spain. ${ }^{10}$ Infectious Diseases Department, Hospital del Mar, Barcelona, Spain. ${ }^{11}$ Department of Preventive Medicine, Hospital Universitari Germans Trias i Pujol, Institut d'Investigació Germans Trias i Pujol, Badalona, Spain. ${ }^{12}$ Fraunhofer Institute for Biomedical Engineering, Ingbert, Germany. ${ }^{13}$ Institució Catalana de Recerca i Estudis Avançats (ICREA), Barcelona, Spain. ${ }^{14}$ These authors contributed equally to this work.

Correspondence: Andreas Meyerhans, ICREA Research Professor at the University Pompeu Fabra, Infection Biology Group, Department of Experimental and Health Sciences, University Pompeu Fabra, Doctor Aiguader, 88, PRBB Building-3rd floor, 08003 Barcelona, Spain. E-mail: andreas.meyerhans@upf.edu

Received: Oct 172014 | Accepted after revision: Dec 042014 | First published online: Feb 052015

Conflict of interest: None declared.

Acknowledgements: Andreas Meyerhans and Irene Latorre were supported by Ministerio de Economía y Competitividad (SAF2010-21336) and ERS/SEPAR-fellowship (LTRF 82-2012), respectively. Jose Domínguez is a researcher from the Miguel Servet programme. Irene Latorre, Jose Domínguez, Maria Luiza de Souza-Galvão, Juan Ruiz-Manzano, Jose Maldonado, Cristina Prat and Andreas Meyerhans are TB-NET members.

\section{References}

1 Walzl G, Ronacher K, Hanekom W, et al. Immunological biomarkers of tuberculosis. Nat Rev Immunol 2011; 11: 343-354.

2 Huang Y, Shen XJ, Zou Q, et al. Biological functions of microRNAs: a review. J Physiol Biochem 2011; 67: 129-139.

3 Leidinger P, Keller A, Backes C, et al. MicroRNA expression changes after lung cancer resection: a follow-up study. RNA Biology 2012; 9: 900-910.

4 Fu Y, Yi Z, Wu X, et al. Circulating microRNAs in patients with active pulmonary tuberculosis. J Clin Microbiol 2011; 49: 4246-4251.

5 Wu J, Lu C, Diao N, et al. Analysis of microRNA expression profiling identifies miR-155 and miR-155* as potential diagnostic markers for active tuberculosis: a preliminary study. Hum Immunol 2011; 73: 31-37.

6 Maertzdorf J, Weiner JIII, Mollenkopf HJ, et al. Common patterns and disease-related signatures in tuberculosis and sarcoidosis. Proc Natl Acad Sci USA 2012; 109: 7853-7858.

7 Miotto P, Mwangoka G, Valente IC, et al. miRNA signatures in sera of patients with active pulmonary tuberculosis. PLoS One 2013; 8: e80149.

8 Zhang X, Guo J, Fan S, et al. Screening and identification of six serum microRNAs as novel potential combination biomarkers for pulmonary tuberculosis diagnosis. PLoS One 2013; 8: e81076.

9 Mestdagh P, Hartmann N, Baeriswyl L, et al. Evaluation of quantitative miRNA expression platforms in the microRNA quality control (miRQC) study. Nat Methods 2014; 11: 809-815.

10 Ma F, Xu S, Liu X, et al. The microRNA miR-29 controls innate and adaptive immune responses to intracellular bacterial infection by targeting interferon-gamma. Nat Immunol 2011; 12: 861-869.

$11 \mathrm{Wu} \mathrm{Z}$, Lu H, Sheng J, et al. Inductive microRNA-21 impairs anti-mycobacterial responses by targeting IL-12 and Bcl-2. FEBS Letters 2012; 586: 2459-2467.

12 Krutzfeldt J, Rosch N, Hausser J, et al. MicroRNA-194 is a target of transcription factor 1 (Tcf1, HNF1alpha) in adult liver and controls expression of frizzled-6. Hepatology 2012; 55: 98-107.

13 Schaale K, Neumann J, Schneider D, et al. Wnt signaling in macrophages: augmenting and inhibiting mycobacteria-induced inflammatory responses. Eur J Cell Biol 2011; 90: 553-559.

14 Bezman NA, Chakraborty T, Bender T, et al. miR-150 regulates the development of NK and iNKT cells. J Exp Med 2011; 208: 2717-2731.

15 Keller A, Leidinger P, Bauer A, et al. Toward the blood-borne miRNome of human diseases. Nat Methods 2011; 8: 841-843. 